\title{
Repurposing: Der schnellere Weg vom Target zur klinisch anwendbaren Substanz
}

Der Erfolg genomweiter Assoziationsstudien hat zu einem verbesserten Verständnis der genetischen Architektur psychiatrischer Erkrankungen geführt und das Interesse der pharmazeutischen Industrie gefördert, die zwischenzeitlich stark zurückgefahrene Entwicklung neuer Wirkstoffe wiederaufzunehmen. Allerdings ist die Medikamentenentwicklung insbesondere für Erkrankungen des Gehirns ein extrem langwieriger und risikobehafteter Prozess. Es kann daher noch viele Jahre dauern, bis die Umsetzung genetisch-neurobiologischer Grundlagenforschung in klinische Anwendungen erfolgt ist. Ein möglicherweise schnellerer Weg, neue pharmakologische Therapien für psychiatrische Erkrankungen in klinischen Anwendungen zu testen, ist das sogenannte „Repurposing“. Darunter versteht man die Wiederverwertung bereits zugelassener Medikamente für sekundäre Indikationen, die u.a. auf bislang unbekannten Wirkmechanismen oder sogenannten „Off-Target“-Effekten beruhen können.
Die zunehmend verbesserte Kenntnis der neurobiologischen Grundlagen psychiatrischer Erkrankungen erlaubt nunmehr die Entwicklung zielgerichteter Messmethoden, um die Wirkung aller klinisch zugelassener Substanzen auf ausgewählte zelluläre Parameter oder Zielmoleküle zu analysieren. In einem „Repurposing“-Ansatz haben wir uns auf den ERBB4-Rezeptor fokussiert, der wesentlich an der Kontrolle des Erregungs-Hemmungs-Gleichgewichts neuronaler Schaltkreise beteiligt ist und einen wichtigen Risikomechanismus psychiatrischer Erkrankungen darstellt. Wir konnten das Diuretikum Spironolacton als neuartigen ERBB4-Modulator identifizieren und kognitive Defizite in passenden Tiermodellen nach Behandlung mit Sprironolacton aufheben. Diese Ergebnisse waren zum einen die Grundlage, um eine klinische Studie mit Patienten, die an Schizophrenie erkrankt sind, zu initiieren und können weiterhin als Bestätigung für den „Repurposing“-Ansatz dienen.
Interessenkonflikte

Mit-Gründer der SYSTASY Bioscience GmbH, die die Patente zur split-Sensor Technology hält; Berater der Boehringer Ingelheim AG \& Co. KG.

\section{Autor}

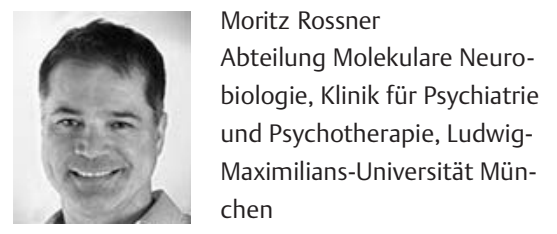

Korrespondenzadresse

Prof. Dr. rer. nat. Moritz Rossner Abteilung Molekulare Neurobiologie Klinik für Psychiatrie und Psychotherapie Ludwig-Maximilians-Universität München Nussbaumstraße 7 80336 München moritz.rossner@med.uni-muenchen.de 